\title{
RÔLE ET பMITATIONS DE LA PLASMONIQUE pour le photovoltaïque solaire
}

Stéphane COLLIN Andrea CATTONI

C2N-CNRS, Marcoussis, France stephane.collin@c2n.upsaclay.fr
À la fin des années 2000, la plasmonique a connu un engouement considérable au sein de la communauté photovoltaïque (PV) : le confinement de la lumière à l'échelle nanométrique devait offrir de nouveaux degrés de liberté dans la conception des cellules solaires, avec l'espoir de diminuer les coûts et d'augmenter les rendements des cellules solaires. Petit bilan après une décennie de recherches intensives.
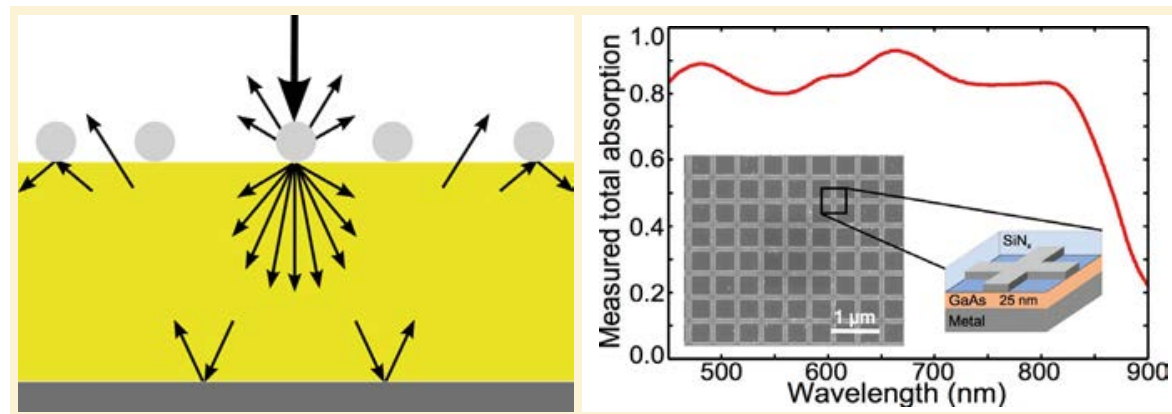

Figure 1. Des nanoparticules métalliques ont été utilisées pour diffuser et piéger la lumière dans des couches de silicium de quelques dizaines de $\mu \mathrm{m}$ d'épaisseur. Avec une couche de semiconducteur de GaAs de seulement $25 \mathrm{~nm}$ d'épaisseur insérée entre un miroir plan et une nano-grille, l'absorption est supérieure à $80 \%$ sur tout le spectre visible. Dans les deux cas, l'absorption parasite dans le métal reste trop élevée pour les applications photovoltaïques. approche consistant à utiliser des nanoparticules métalliques pour concentrer et diffuser la lumière dans des couches semiconductrices très fines a été rapidement abandonnée:l'effet est faible, et les pertes parasites dans les particules sont rédhibitoires. Une seconde approche vise à utiliser l'absorption parfaite de la lumière dans un résonateur plasmonique de très petite dimension, qui est bien établie, puis à rendre les structures multi-résonantes pour élargir la gamme spectrale. Il est ainsi possible d'absorber $80 \%$ de la lumière incidente du spectre visible dans seulement $25 \mathrm{~nm}$ de GaAs [2]. Mais les pertes parasites sont encore trop importantes avec les matériaux utilisés actuellement (métaux nobles).

Une des conclusions actuelles, très positive, est quela plasmonique permeten effet d'absorber efficacement la lumière dans un volume de très faible dimension (voir $f$ gure 1). Mais deux autres conclusions, plus nuancées, sontquel'échelle nanométrique ne semble pas adaptée à la conception d'une cellule solaire, et que les pertes parasites dans le métal semblent rédhibitoires.

Aujourd'hui, la recherche sur les cellules solaires ultrafines reste très active. Les effets plasmoniques sont évités, mais la nanophotonique est très présente dans les travaux en cours pour piéger la lumière dans des couches planaires nanostructurées ou dans des nanofils. Des cellules solaires de seulement quelques centaines de nanomètres d'épaisseur voient le jour avec des rendements approchant le seuil des $20 \%$ : de toute évidence, la nanophotonique a encore beaucoup à apporter au photovoltaïque!

\section{POUR EN SAVOIR PLUS}

[1] H.A. Atwater, A. Polman, Plasmonics for improved photovoltaic devices, Nature Materials 9, 205 (2010)

[2] I. Massiot et al., Metal nanogrid for broadband multiresonant light-harvesting in ultrathin GaAs layers, ACS Photonics 1, 878 (2014) doivent rester marginales pour préserver l'efficacité des cellules. Une première 